\title{
Hierarchically Porous Hydroxyapatite Hybrid Scaffold Incorporated with Reduced Graphene Oxide for Rapid Bone Ingrowth and Repair
}

Kai Zhou ${ }^{\dagger}, z^{\prime}$ Peng Yu, ${ }^{*}$ Xiaojun Shi, ${ }^{\dagger}$ Tingxian Ling, ${ }^{\dagger}$ Weinan Zeng, ${ }^{\dagger}$ Anjing Chen ${ }^{\dagger}$ Wei Yang, ${ }^{\ddagger, *}$ Zongke Zhou ${ }^{\dagger}$ **

${ }^{\dagger}$ Department of Orthopaedics, West China Hospital of Sichuan University, Chengdu, 610041, Sichuan, China

${ }^{\ddagger}$ College of Polymer Science and Engineering, Sichuan University, State Key Laboratory of Polymer Materials Engineering, Chengdu, 610065, Sichuan, China ${ }^{\text {z }}$ State Key Laboratory of Biotherapy and Cancer Center, West China Hospital, Sichuan University and Collaborative Innovation Center, Chengdu, 610041, China

Corresponding Authors

E-mails: weiyang@scu.edu.cn (W Yang) and zhouzongke@scu.edu.cn (Z Zhou)

Tel: 0086-028-85422570; Fax: 0086-028-85423438 


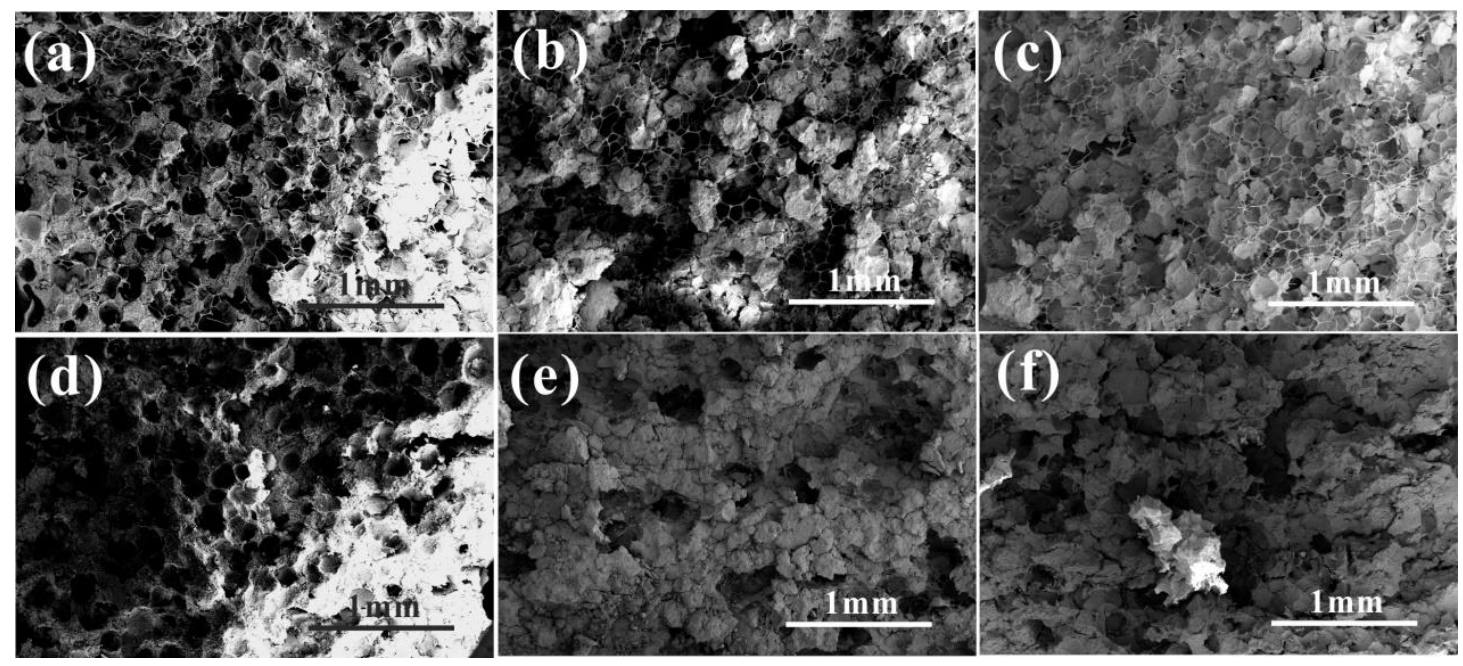

Figure S1. SEM images of before sintering (a) HA-6 (b) HA-6-CS and (c) HA-6-D;

SEM images of (d) HA-6 (e) HA-6-CS and (f) HA-6-D
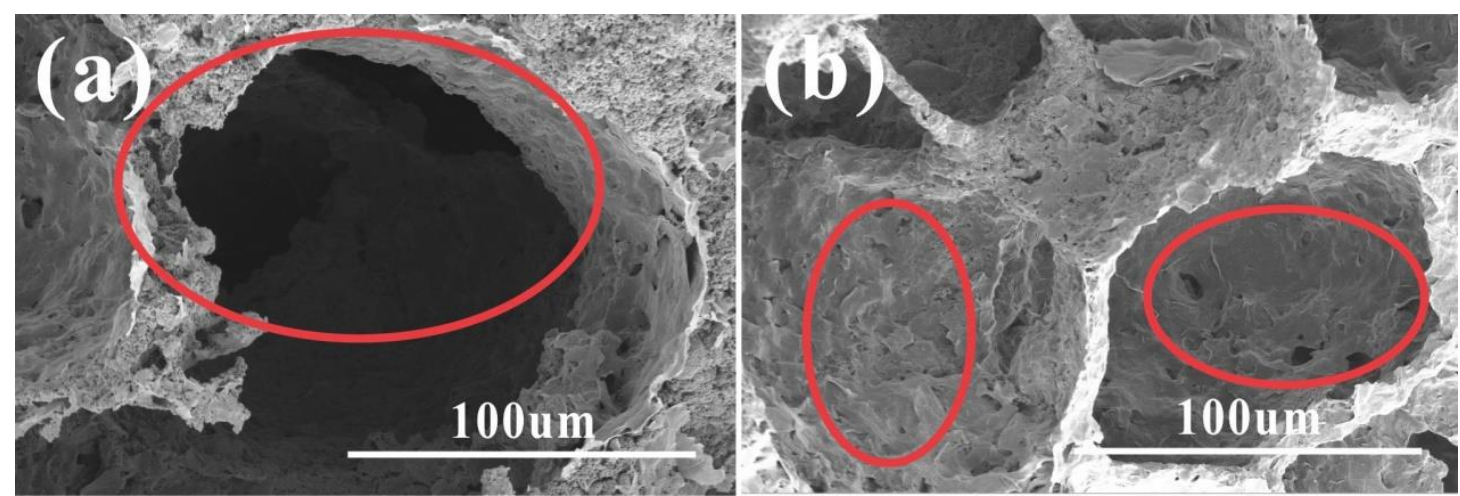

Figure S2. SEM images of (a) H/G-6/0.3\% and (b)H/G-6/0.9\%.

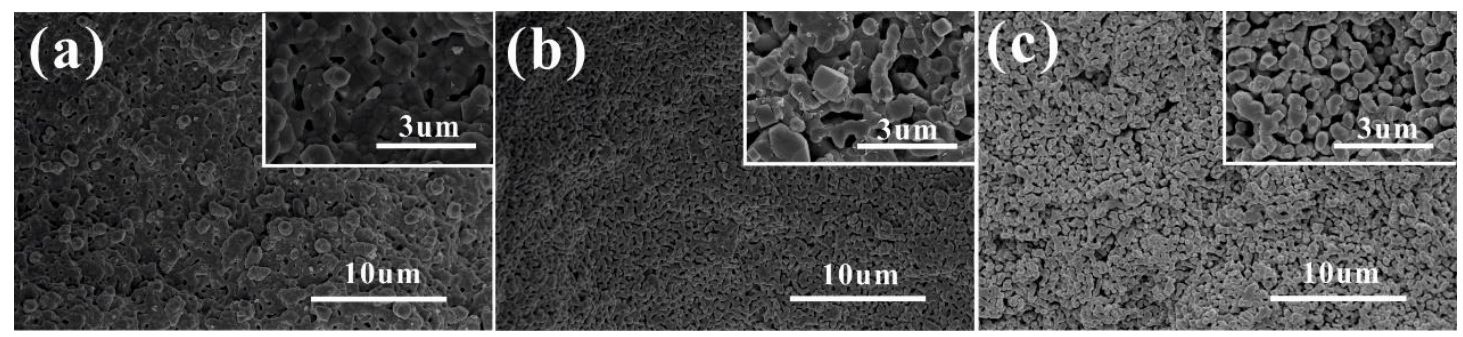

Figure S3. SEM images of (a) H/G-6/0.3\% (b) HA-6-D (c) HA-6-CS. 


\section{Surgical Procedures}

The surgical operation was performed under general anesthesia by abdominal injection of $10 \%$ chloral hydrate $(0.2 \mathrm{ml} / 100 \mathrm{~g})$. Each rat was immobilized in a lateral position and its hind leg was shaved and swabbed twice with iodophor. Afterward, an outer thigh lateral approach with a $25 \mathrm{~mm}$ incision was performed and the deep fascia was divided. By blunt dissection of the interval of the quadriceps muscles, the femur was exposed and the periosteum was stripped carefully. The segmental defects were stabilized with the RatFix ${ }^{\circledR}$ (RISystem AG, Davos, Switzerland) osteosynthesis system. ${ }^{1}$ A plate was first fixed by 4 screws at the mid-shaft femur where a $6 \mathrm{~mm}$ critical-size segmental diaphyseal bone defect was then created by fretsaw (Figure S1). According to the group, it is planned to implant the materials prepared in advance in the bone defect. The wound was irrigated and closed carefully. Following the surgeries, 400,000 units of penicillin were intraperitoneal injection for 3 days to prevent infection. After the operation, the animals were housed in ventilated rooms and allowed for full weight-bearing activities. 

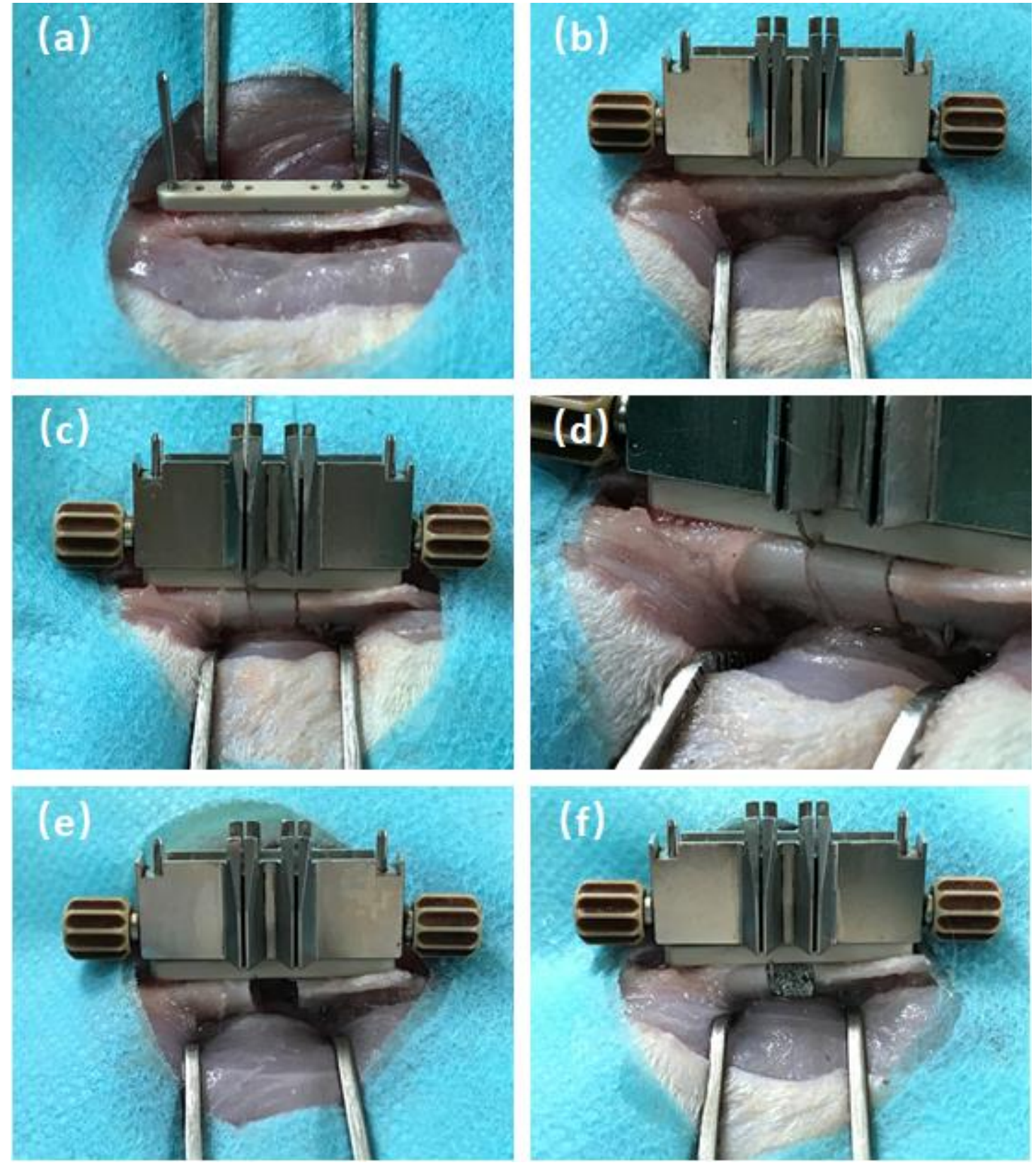

Figure S4. Establishment of a rat femoral diaphyseal defect model with RatFix ${ }^{\circledR}$ osteosynthesis system. (a) Fully expose the femur, place the RISystem ${ }^{\circledR}$ plate, and fix it with screws; (b) Installation of osteotomy guide; (c), (d) Using wire saw to osteotomy; (e) Osteotomy completed; (f) Fill the material into the defect. 

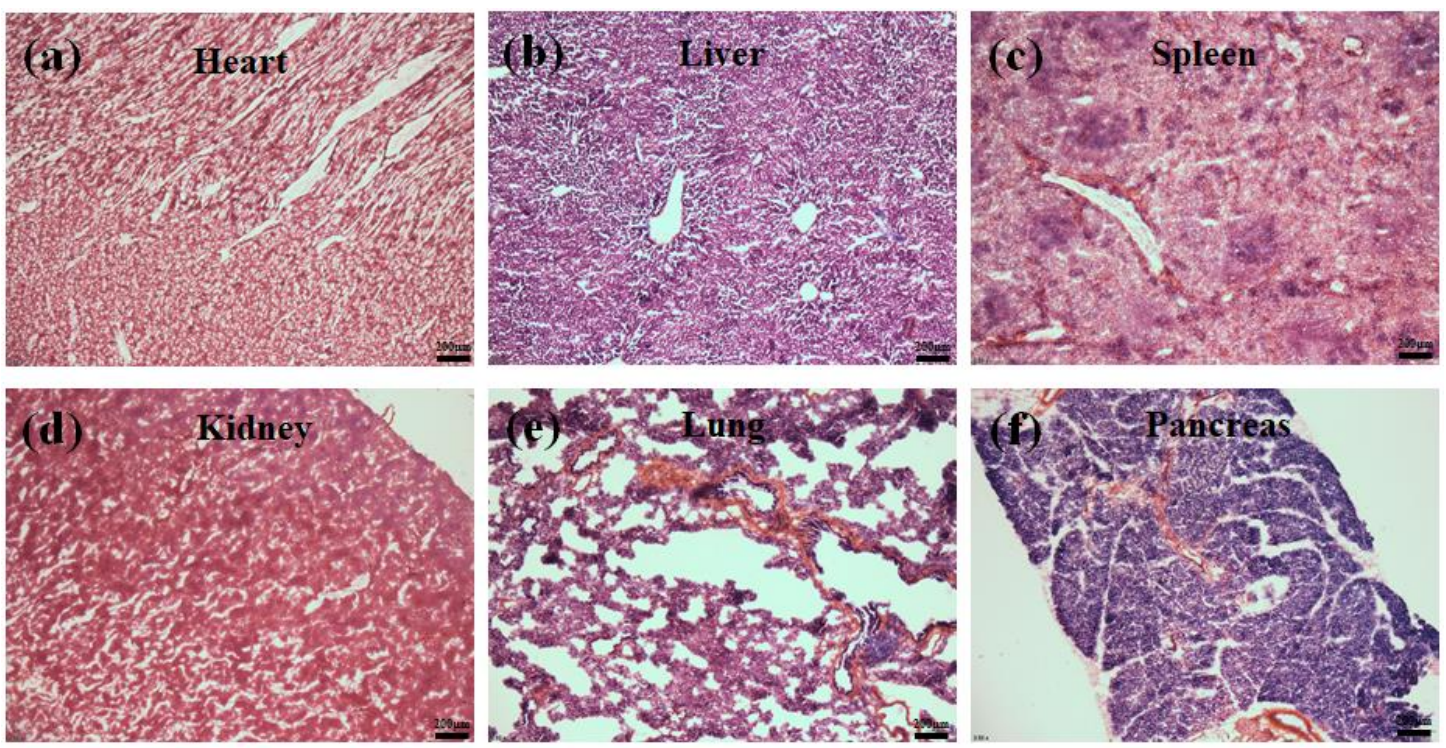

Figure S5. HE staining of rat heart, liver, spleen, kidney, lung and pancreas after implantation of HA/rGO-6/0.3 material for half an year. No obvious abnormalities in all observed organizations.

Table S1. Primer pairs used in real-time PCR analysis ( $\mathrm{F}=$ forward, $\mathrm{R}=$ reverse).

\begin{tabular}{|c|c|c|}
\hline Gene & & Primers \\
\hline \multirow{3}{*}{$\beta$-actin } & $\mathrm{F}$ & GTGACGTTGACATCCGTAAAGA \\
\hline & & \\
\hline & $\mathrm{R}$ & GTAACAGTCCGCCTAGAAGCAC \\
\hline \multirow{3}{*}{ ALP } & $\mathrm{F}$ & TCGGGACTGGTACTCGGATAAC \\
\hline & & \\
\hline & $\mathrm{R}$ & GTTCAGTGCGGTTCCAGACATAG \\
\hline \multirow{3}{*}{ BMP-2 } & $\mathrm{F}$ & CGAATTTGAGTTGAGGCTGCTC \\
\hline & & ССССТТТТТС САСТСАТСТСТ \\
\hline & & \\
\hline \multirow{3}{*}{$\mathrm{OCN}$} & $\mathrm{F}$ & TTTCTGCTCACTCTGCTGACC \\
\hline & & \\
\hline & $\mathrm{R}$ & CAGCACAACTCCTTCCT ACCA \\
\hline
\end{tabular}


OPN

$\mathrm{R}$

F

Runx2

COL-I
$\mathrm{R}$

F

$\mathrm{R}$
CTGCCCTTTCCGTTGTTGTC

AGCGGACGAGGCAAGAGTTT

AGGCGGGACACCT ACTCTCAT A

CATGTTCAACCCAGAGGAGCG

GGTCCATGTAGGCTACGCTGTT 


\section{REFERENCE}

(1) Sebald, H. J.; Klenke, F. M.; Siegrist, M.; Albers, C. E.; Sebald, W.; Hofstetter, W. Inhibition of Endogenous Antagonists with an Engineered BMP-2 Variant Increases BMP-2 Efficacy in Rat Femoral Defect Healing. Acta Biomater. 2012, 8, 3816-3820. 